\title{
INFLUENCE OF FREE CARRIER CONCENTRATION ON NONLINEAR ABSORPTION OF $n$-TYPE ZnSe CRYSTALS
}

\author{
B. Sahraoui*, R. Chevalier, X. Nguyen Phu, G. Rivoire
}

Laboratoire POMA, Propriétés Optiques des Matériaux et Applications

Université d'Angers

2, boulevard Lavoisier, 49045, Angers cedex, France

\section{AND W. BAEA}

Institute of Physics, N. Copernicus University, Grudziądzka 5, 87-100 Toruń, Poland

The dependence of nonlinear absorption at $532 \mathrm{~nm}$ of $n$-type $\mathrm{ZnSe}$ crystals upon annealing temperature and free carrier concentration is reported. The nonlinear optical absorption as well as the efficiency of degenerate four wave mixing of $\mathrm{ZnSe}$ are investigated. It is found that the magnitude of the nonlinear absorption decreases with an increase in the electron concentration. The nonlinear refractive index change is estimated.

PACS numbers: $78.20 . \mathrm{Jq}, 78.30 \mathrm{Hv}$

\section{Introduction}

Wide-gap semiconducting materials are being widely studied as potential components of various optical systems, especially for light-emitting diodes and lasers which operate in the blue-green region of the spectrum, and nonlinear optical devices [1-5]. Knowledge of the absorption coefficients and refractive indexes of crystals under high excitation is especially important in the design and analysis of laser structures and waveguiding devices using these semiconductors in the visible and infrared range. Although the optical properties of $\mathrm{ZnSe}$ crystals have been well established recently, little is known about their nonlinear properties versus concentration of free carriers. The nonlinear optical properties of $\mathrm{ZnSe}$ crystals with different free carrier concentrations are studied here using picosecond pulses from a mode locked Quantel YAG laser operating at $532 \mathrm{~nm}$. The pulses are nearly Fourier transform limited and the laser operates at the $1 \mathrm{~Hz}$ repetition rate.

*Also in the Institute of Physics, N. Copernicus University, Toruń, Poland. 


\section{Preparation of the samples}

Intentionally undoped zinc selenide ( $\mathrm{ZnSe}$ ) crystals were grown from the melt by the modified high-pressure Bridgman method under argon over pressure of $11 \mathrm{MPa}$ using $\mathrm{ZnSe}$ powder (6N Koch-Light) as a starting material. The crystals were cut into $1 \mathrm{~mm}$ thick plates parallel to (111) crystallographic plane and annealed in liquid zinc at different temperatures in the range $750-950^{\circ} \mathrm{C}$. After this process, the samples are mechanically polished and chemically etched in the mixture of $\mathrm{K}_{2} \mathrm{CrO}_{7}: \mathrm{H}_{2} \mathrm{SO}_{4}: \mathrm{H}_{2} \mathrm{O}$ in proportion $3: 2: 1$, followed by a treatment in $\mathrm{CS}_{2}$ and boiling $14 \mathrm{n} \mathrm{NaOH}$ solution. The final thickness of ZnSe crystals in the measurement is $0.73 \mathrm{~mm}$. The measurements of the resistivity, Hall mobility and carrier concentration were performed by the Van der Pauw method using indium dots as contacts. The influence of annealing temperature on the electron concentration was presented in paper [6] and is displayed in Table.

TABLE

The values of linear and nonlinear absorption coefficients $\alpha$ and $\beta$ in $\mathrm{ZnSe}$ for different annealing temperatures $T$ and free carriers concentration $n$.

\begin{tabular}{c|c|c|c|c}
\hline \hline Ref. crystals & $T\left[{ }^{\circ} \mathrm{C}\right]$ & $n\left[\mathrm{~cm}^{-3}\right]$ & $\alpha\left[\mathrm{cm}^{-1}\right]$ & $\beta\left[\mathrm{cm} \mathrm{GW}^{-1}\right]$ \\
\hline 1 & 760 & $1.5 \times 10^{15}$ & 15 & 27 \\
2 & 780 & $3.2 \times 10^{15}$ & 13.2 & 24.6 \\
3 & 830 & $1.5 \times 10^{16}$ & 9.3 & 17.3 \\
4 & 870 & $7.4 \times 10^{16}$ & 5 & 14.9 \\
5 & 890 & $1.3 \times 10^{17}$ & 3.3 & 13.7 \\
6 & 920 & $2.9 \times 10^{17}$ & 2.1 & 12.3
\end{tabular}

\section{Results and discussion}

The following experiments have been performed:

- Pump transmission versus pump fluence: in this case only one beam is sent in the medium.

- Degenerate four wave mixing measurements (DFWM): the signal beam intensity is measured as a function of the exciting beam intensities.

\subsection{Nonlinear transmission}

The absorption is characterized by the transmission of $\mathrm{ZnSe}$ as a function of input intensity. In ZnSe, two-photon absorption is observed at $532 \mathrm{~nm}$ as the incident photon energy is lower than the direct band gap energy $E_{\mathrm{g}}$ but greater than $E_{\mathrm{g}} / 2$ [7]. The linear absorption is due, among other reasons, to the impurity levels in the band gap. If the beam $\langle 1\rangle$ is sent alone in the medium, its transmission can be expressed as follows: 


$$
T=\frac{I_{1}(l)}{I_{1}(0)}=\frac{\alpha \exp (-\alpha l)}{\alpha+\beta I_{1}(0)[1-\exp (-\alpha l)]}
$$

where $l$ is the sample length.

To deduce the absorption coefficients $\alpha$ and $\beta$, we perform for all crystals studied the best fit of the experimental data (Fig. 1) using Eq. (1). The obtained results show that the magnitude of the nonlinear absorption decreases with an increase in the electron concentration (Table). Coming out from the first-order perturbation approach which takes into account electron-phonon interaction, an increase in free electron concentration should lead to decrease in corresponding nonlinear optical absorption response.

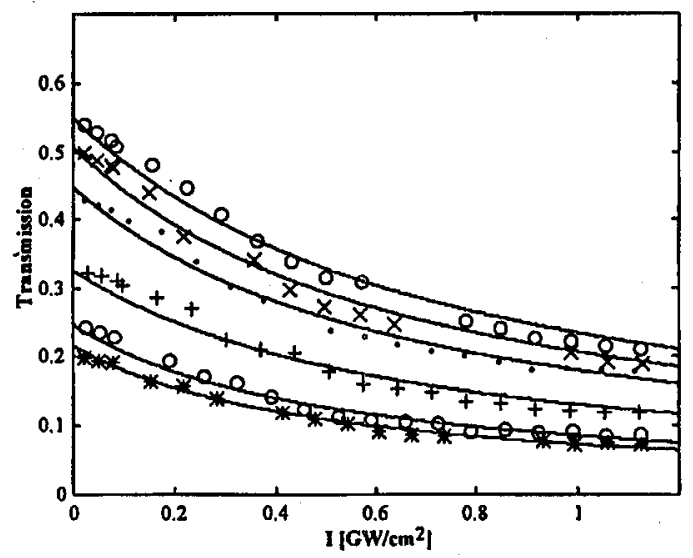

Fig. 1. The nonlinear transmission as a function of the incident beam intensity $I_{1}$ in $\mathrm{ZnSe}$ for different annealing temperatures $\left({ }^{*} 760^{\circ} \mathrm{C}, \circ 780^{\circ} \mathrm{C},+830^{\circ} \mathrm{C}, \bullet 870^{\circ} \mathrm{C}\right.$, $\times 890^{\circ} \mathrm{C}$, and $\circ 920^{\circ} \mathrm{C}$ ). All samples reveal a strong nonlinear (two-photon) absorption of light. For all sets of the experimental data we perform the best fit of the data using Eq. (1) to extract the $\alpha$ and $\beta$ values.

\section{2. $D F W M$}

The DFWM experiment with the basic geometry illustrated in Fig. 2 is used to measure the DFWM efficiency of $\mathrm{ZnSe}$ crystals. The incident wave intensities verify the relations: $I_{1}(z=0)=I_{2}(z=l)$ and $I_{3}=10^{-2} I_{1}$. The angle $\theta$ between

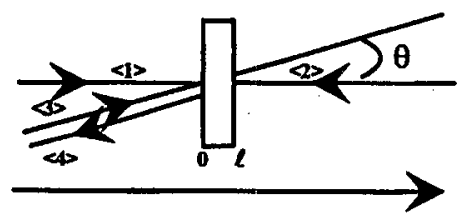

Fig. 2. Geometry of degenerate four wave mixing experiment. 


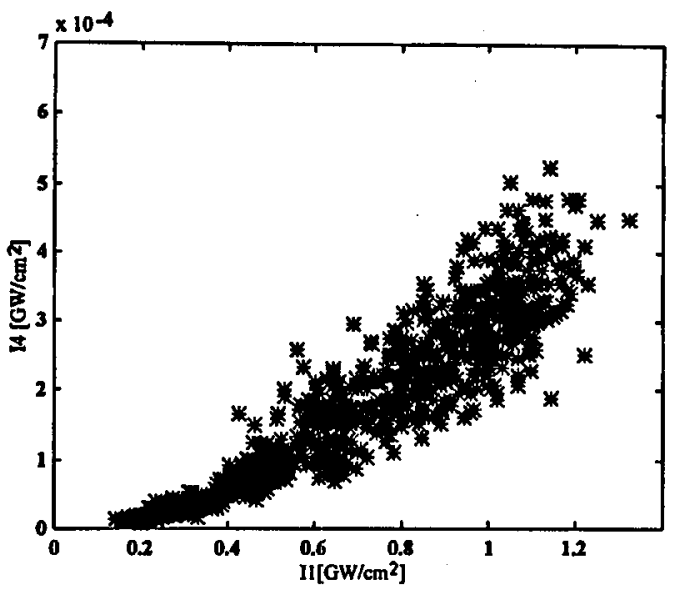

Fig. 3. The fourth beam intensity $I^{\langle 4\rangle}$ with respect to the pump intensity $I^{\langle 1\rangle}$ for $\mathrm{ZnSe}$. * represent the experimental data for $n$-type $\mathrm{ZnSe}$ annealed at the temperature $870^{\circ} \mathrm{C}$.

the beams $\langle 1\rangle$ and $\langle 3\rangle$ in air is $12^{\circ}$. The beam $\langle 4\rangle$ intensity is measured as a function of the exciting beam intensity for all crystals studied. As an example we present in Fig. 3 the experimental data for $\mathrm{ZnSe}$ crystal annealed at $870^{\circ} \mathrm{C}$. The phase conjugation efficiency $R$ is calculated from the propagation equations [8] of the four beams in interaction, using the slowly varying amplitude approximation and taking in account linear and nonlinear absorption and the transformation from the crystallographic axis to laboratory axis. The phase conjugation efficiency $R$ can be expressed as follows:

$$
R=\frac{I_{4}(0)}{I_{3}(0)}=\frac{K^{2}}{[q \operatorname{coth}(q l)-\Psi / 2]^{2}}
$$

where

$$
\begin{aligned}
& q^{2}=(\Psi / 2)^{2}-K^{2}, \quad K^{2}=\left(\frac{4 \pi H}{n c}\right)\left({\chi^{\prime}}^{2}+\chi^{\prime \prime 2}\right) I_{1} I_{2} \\
& H=\frac{12 \pi^{2}}{n \lambda} \text { and } \Psi=-\alpha-2 \beta\left(I_{1}+I_{2}\right)
\end{aligned}
$$

$\chi^{\prime}$ and $\chi^{\prime \prime}$ are the real and imaginary parts of the sum of the four independent non-zero components of the susceptibility tensor $\chi_{x x x x}+\chi_{x x y y}+\chi_{x y x y}+\chi_{x y y x}$. All crystals studied display relatively strong absorption which means $\Psi^{2}-4 K^{2}>0$. The value of $\chi^{\prime \prime}$ is deduced from $\beta$, and thus $\chi$ can be extracted from the measured values of $R$ compared to formula (2). The estimated value of the real part of $\chi^{(3)}$ for $\mathrm{ZnSe}$ annealed at $870^{\circ} \mathrm{C}$ is $(5.7 \pm 1) \times 10^{-12}$ (e.s.u. system) (nearly 3 times the $\mathrm{CS}_{2}$ value). The value $\chi$ of increases with $n$. To deduce the sign of real part of $\chi^{\langle 3\rangle}$ we have performed supplementary tests concerning self-defocusing of a single beam travelling through $\mathrm{ZnSe}[9,10]$. We have found that for $\mathrm{ZnSe}$ the sign of real part of $\chi^{\langle 3\rangle}$ is negative. 


\section{Acknowledgment}

This work was supported in part by the Committee for Scientific Research grant PBZ 101.01 (W.B.). The authors wish to thank Dr. F. Firszt for annealing of the $\mathrm{ZnSe}$ samples.

\section{References}

[1] H.J. Lozykowski, V.K. Shastre, J. Lumin. 48\&49, 740 (1991).

[2] K.H. Pantke, L. Broser, Phys. Rev. B 48, 11752 (1993).

[3] H. Joen, J. Ding, A.V. Nurmikko, W. Xie, M. Kobayashi, R.L. Gunshor, Appl. Phys. Lett. 75, 892 (1992).

[4] S. Ohtsuka, T. Koyama, K. Tsunetomo, H. Nagata, S. Tanaka, Appl. Phys. Lett. 61, 2953 (1992).

[5] H. Morkoc, S. Strite, G.B. Gao, M.E. Lin, Sverdlov, M. Burns, Appl. Phys. Lett. 76, 1363 (1994).

[6] N. Kozielski, M. Drozdowski, W. Bala, F. Firszt, Acta Phys. Pol. A 73, 369 (1988).

[7] J.H. Bechtel, W. Smith, Phys. Rev. B 13, 3515 (1976).

[8] B. Sahraoui, M. Sylla, J.P. Bourdin, G. Rivoire, J. Zaremba, T.T. Nguyen, M. Sallé, J. Mod. Opt. 42, 2095 (1995).

[9] M. Sheik-Bahae, Ali A. Said, Tai-Huei Wei, D.J. Hagan, E.W. Van Stryland, J. Quantum Electron. 26, 760 (1990).

[10] N.P. Xuan, J.L. Ferrier, J. Gazengel, G. Rivoire, Opt. Commun. 46, 5 (1983). 